308

\title{
COMPOSIÇÃO IDEAL DA SOLUÇÃO FILMOGÊNICA ADICIONADA DE PREBIÓTICO, APLICADA EM UVAS 'THOMPSON'
}

\author{
IURI MIRA², CELSO DUARTE CARVALHO FILHO ${ }^{3}$, DENISE NUNES VIOLA ${ }^{4}$
}

RESUMO-A aplicação de coberturas comestíveis em frutas e hortaliças deve ser precedida de testes que busquem a formulação que melhor se adapta àquele produto. $\mathrm{O}$ objetivo deste trabalho foi determinar a composição ideal da cobertura comestível à base de cera de carnaúba adicionada de prebiótico a ser aplicada em uvas variedade Thompson, armazenadas sob refrigeração. Após a seleção, os frutos foram higienizados e procedeu-se à aplicação de soluções aquosas de cera de carnaúba adicionadas de Fruto-oligossacarídeo (FOS). Foram testadas treze formulações, utilizando as combinações de $50 ; 33 ; 25$ e $0 \%$ (v/v) de cera de carnaúba e $30 ; 20 ; 10$ e $0 \%$ do FOS (p/v). Durante 25 dias, foram avaliados parâmetros de textura, físicoquímicos e físicos. Os parâmetros de textura avaliados sofreram alterações durante o período do estudo, mas sem evidências de terem sofrido interferência das coberturas aplicadas. As formulações que se mostraram mais promissoras para futuras aplicações comerciais foram dos tratamentos B ( $50 \%$ de cera, $20 \%$ de FOS), K ( $25 \%$ de cera, $10 \%$ de FOS) e L ( $25 \%$ de cera, $0 \%$ de FOS).

Termos para indexação: parâmetros físico-químicos, avaliação de textura, cobertura comestível, pós-colheita.

\section{IDEAL COMPOSITION OF FILM SOLUTION ADDED TO PREBIOTIC, APPLIED IN 'THOMPSON' GRAPES}

\begin{abstract}
The application of edible coatings for fruits and vegetables should be preceded by tests that seek the formulation that best fits that product. The objective of this study was to determine the ideal composition of edible coating based on carnauba wax added to prebiotic to be applied on grapes Thompson variety, stored under refrigeration. After selection, the fruits were cleaned and proceeded to apply the aqueous solutions of carnauba wax added to fructooligosaccharides (FOS). Thirteen formulations were tested, using the combinations 50,33, 25 and $0 \%(\mathrm{v} / \mathrm{v})$ of carnauba wax and 30, 20, 10 and $0 \%$ of the FOS (w/v). For 25 days, texture, physicochemical and physical parameters were evaluated. The texture parameters were changed during the study period, but no evidence have been influenced the coverage applied. The tested edible coatings did not alter the parameters evaluated of the Thompson grapes. The formulation which showed the most promising for future commercial applications were the treatment B ( $50 \%$ wax, $20 \% \mathrm{FOS}), \mathrm{K}(25 \%$ wax, $10 \%$ FOS $)$ and L (25\% wax, $0 \%$ FOS).
\end{abstract}

Index terms: physicochemical parameters, evaluation of texture, edible coating, postharvest.

1'(Trabalho 349-13). Recebido em: 25-09-2013. Aceito para pubilcação em: 16-03-2015.

${ }^{2}$ Aluno do Programa de Pós-Graduação em Ciência de Alimentos da FacFar-UFBA. Rua Barão de Geremoabo, S/N, Ondina. CEP: 40170-115. Salvador-BA. E-mail: iurimira@gmail.com

${ }^{3}$ Prof. Dr. do Programa de Pós-Graduação em Ciência de Alimentos. Fac. Farmácia-UFBA. celsodc@ufba.br

${ }^{4}$ Prof. Dr. do Programa de Pós-Graduação em Ciência de Alimentos. Fac. Farmácia-UFBA. viola@ufba.br

Rev. Bras. Frutic., Jaboticabal - SP, v. 37, n. 2, p. 308-317, Junho 2015 


\section{INTRODUÇÃO}

A uva (Vitis vinifera) é fruta de diversificada variedade e forma de consumo (MATTIUZ et al., 2009; MELLO, 2011b), sendo a produção de uvas finas de mesa uma importante atividade econômica no Brasil (MELLO, 2011a). No mercado brasileiro de uvas de mesa, seguindo uma tendência mundial, percebe-se uma exigência cada vez maior dos consumidores por frutas de melhor qualidade, não somente quanto ao aspecto, mas também ao sabor, aroma e consistência, além de uma preferência por uvas sem sementes ou apirênicas, a exemplo da variedade Thompson (LULU et al., 2005).

A uva é uma fruta não climatérica, devendo ser colhida apenas quando atingir as condições apropriadas para o consumo. Por outro lado, ela é considerada uma fruta de alta perecibilidade, e mesmo com os avanços tecnológicos, as perdas pós-colheita do produto, estimadas entre 20 e $95 \%$, ainda causam grandes prejuízos aos viticultores (ALBERTINI et al., 2009; MATTIUZ et al., 2009; LIMA, 2010; DEL NOBILE et al., 2009). Os principais problemas de conservação pós-colheita das uvas de mesa estão relacionados às alterações de textura e à ocorrência de danos mecânicos, que comprometem a aceitação destes frutos pelos consumidores (MATTIUZ et al., 2009; DEL NOBILE at al., 2009).

Devido à demanda por alimentos de alta qualidade e preocupações ambientais sobre o descarte de materiais não renováveis, existe um crescente interesse no desenvolvimento de biofilmes comestíveis ou degradáveis biologicamente (FAKHOURI; GROSSO, 2007; MOTA et al., 2006; VU et al., 2011). Coberturas comestíveis (Edible coatings) são, para a maioria dos autores, substâncias aplicadas na superfície de alimentos de modo que o produto final está pronto para o consumo (BALDWIN et al., 2011). Os filmes, além de constituírem-se uma barreira efetiva à perda de vapor d'água e troca gasosa apresentam-se como materiais de embalagem biodegradáveis que podem ajudar na proteção contra danos mecânicos e na contaminação microbiana, sendo mais frequente seu uso em frutas e hortaliças (ALLEONI et al., 2006; CARVALHO FILHO et al., 2006; VU et al., 2011).

Lipídeos e ceras tendem a ser mais permeáveis a gases, mas apresentam a melhor barreira a vapor d'água e incluem cera de carnaúba, candelilla e farelo de arroz (BALDWIN et al., 2011). A cera de carnaúba vem sendo utilizada como cobertura comestível no revestimento de diversas frutas, como maracujáamarelo e cereja (MOTA et al., 2006; CARVALHO FILHO et al., 2006).
As coberturas comestíveis podem ser utilizadas para carrear ingredientes funcionais, como fungicidas, antioxidantes e agentes de firmeza, melhorando a estabilidade microbiológica, a aparência e a textura do produto coberto (ZHAO, 2011; VU et al., 2011). Podem também ser incorporados nestas coberturas compostos com ação prebiótica, a exemplo dos fruto-oligossacarídeos (FOS).

Os FOS são açúcares não convencionais, não metabolizados pelo organismo humano e não calóricos (HARTEMINK et al., 1997; BERTÉ et al., 2011; ROBERFROID et al., 2010). As boas características físicas dos FOS, como apresentar alta solubilidade e não cristalização em solução, permitem sua aplicação em diversas áreas, com uso em formulações diversas, como em alimentos funcionais (PASSOS; PARK, 2003).

O uso de atmosfera modificada, como a aplicação de coberturas comestíveis em alimentos não processados, deve ser precedido de testes que demonstrem a formulação que melhor se adapte àquele produto, visto que as frutas e hortaliças têm metabolismo variado de acordo com a espécie, variedade cultivada e condições de manejo no campo. Com base nestas particularidades, o objetivo deste trabalho foi determinar a composição ideal da solução filmogênica adicionada de prebiótico aplicada em uvas (Vitis vinifera), variedade Thompson, através da análise dos parâmetros físicos, físico-químicos e mecânicos.

\section{MATERIAL E MÉTODOS}

\section{Aquisição das Uvas}

Foram utilizados $20 \mathrm{~kg}$ de uvas, variedade Thompson Seedless. As uvas, obtidas da safra agosto/ outubro de 2012, foram colhidas no dia anterior ao início da pesquisa, diretamente do produtor, no município de Juazeiro - BA, região do Vale do São Francisco.

As uvas permaneceram em suas embalagens primárias e foram transportadas em caixas isotérmicas à temperatura de aproximadamente $5^{\circ} \mathrm{C}$, monitoradas por termômetro até o laboratório de processamento e análises, em Salvador - BA.

\section{Pré-Tratamento dos Frutos}

Os cachos de uva foram inicialmente lavados em água corrente para retirada de sujidades físicas. Após a lavagem, foi realizada a separação das bagas de uva do engaço, utilizando tesoura específica, mantendo o pedúnculo fixado à baga. Houve seleção das bagas, na qual foram considerados o tamanho, a uniformidade de cor e a integridade dos frutos de maneira a estabelecer homogeneidade entre eles. 
As bagas foram então higienizadas por imersão em solução de hipoclorito de sódio, na concentração de $100 \mathrm{ppm}$ por 15 minutos e mantidas em ambiente climatizado até a secagem completa.

Elaboração das Coberturas Comestíveis

Foi utilizada emulsão de cera de carnaúba JVC WAX 36 (36\% de cera de carnaúba), da empresa Química JVC LTDA. (Feira de Santana, Brasil) como matriz da solução filmogênica e o fruto-oligossacarídeo ORAFTI GR $(96,4 \%$ de oligofrutose) da empresa Clariant (Oreye, Bélgica) como prebiótico.

Com base em testes preliminares, recomendações de fornecedores e trabalhos publicados (CARVALHO FILHO et al., 2006; DEL NOBILE et al., 2009), foi realizado delineamento experimental fatorial do tipo $4^{2}$, com dois fatores, concentração da emulsão de cera de carnaúba e concentração do prebiótico, em quatro níveis cada, a fim de obter a melhor formulação. As concentrações testadas foram de $50 ; 33 ; 25 \mathrm{e} 0 \%(\mathrm{v} / \mathrm{v})$ para a cera de carnaúba e 30; 20; 10 e $0 \%$ para o prebiótico $(\mathrm{p} / \mathrm{v})$.

Devido à impossibilidade de formação de solução filmogênica, apenas com o prebiótico e água, fez-se necessária a exclusão dos tratamentos com $0 \%$ de cera $(0: 30 ; 0: 20$ e $0: 10$ de cera:prebiótico), formando um fatorial incompleto, com 13 tratamentos ao invés dos 16, conforme apresentado na Tabela 1.

As soluções filmogênicas foram obtidas por solubilização direta do prebiótico em água ultrapura, à temperatura ambiente. Foi realizada agitação manual por dez minutos até a completa solubilização do FOS. Em seguida, foi adicionada a cera de carnaúba, sob agitação manual, à temperatura ambiente, e avolumada a solução com água ultrapura. A espuma formada foi retirada com auxílio de espumadeira.

\section{Aplicação das Coberturas}

As uvas mantiveram-se imersas durante dez segundos em sua respectiva solução filmogênica, em seguida permaneceram suspensas pelo pedúnculo, em ambiente climatizado, até secagem completa. No tratamento-controle, as frutas foram imersas em água ultrapura por igual período e submetidas à secagem, conforme as amostras tratadas.

Após a secagem, os frutos foram acondicionados em cestas de videiras plásticas de polietileno tereftalato (PET), perfuradas, identificadas de acordo com o tratamento recebido e mantidas sob refrigeração $\left(2\right.$ a $\left.8^{\circ} \mathrm{C}\right)$. Cada tratamento foi distribuído em três cestas, sendo uma para análise de perda de massa e duas destinadas para testes destrutivos, como avaliação da resistência mecânica, teores de sólidos solúveis e acidez total titulável.

\section{das Uvas \\ Propriedades Físicas e Físico-Químicas}

As análises de perda de massa, teores de sólidos solúveis (SS) e acidez total titulável (ATT) e de propriedades mecânicas ocorreram em intervalos de três dias, tendo início no dia seguinte à aplicação das coberturas. A aferição de comprimento e diâmetro das bagas, realizada com paquímetro, a avaliação de impressão e a análise de cor ocorreram em momento único, no dia seguinte à aplicação das coberturas.

As cestas de videiras, destinadas à avaliação da perda de massa, foram identificadas e pesadas em balança analítica. As taxas de perda de massa diária e acumulada foram calculadas tomando como referência a massa inicial de cada cesta de videira, no dia seguinte à aplicação das coberturas.

Oito bagas de cada tratamento foram utilizadas para a avaliação do aspecto global, utilizando escala hedônica de nove pontos, correspondendo o valor 1 a "desgostei muitíssimo", e o valor 9, a "gostei muitíssimo" para os parâmetros Tonalidade de Cor, Brilho e Aparência Global, esta última relacionada à percepção de irregularidades na superfície das bagas. Nestas oito bagas, foi realizada também análise de cor, sendo estas aferições realizadas em triplicata, diretamente sobre a superfície da fruta íntegra. Os parâmetros L*, a* e b* foram determinados utilizando-se de colorímetro marca Konica Minolta, modelo CR-400 (Ramsey, EUA).

A determinação de sólidos solúveis foi realizada utilizando-se do método 313/IV do INSTITUTO ADOLFO LUTZ (2005). A partir do homogeneizado de oito bagas de uva de cada tratamento, foram realizadas leituras, em octuplicata, utilizando-se de refratômetro marca QUIMIS, modelo Q-767-1 (Diadema, Brasil).

A acidez foi determinada utilizando-se de $4 \mathrm{~g}$ de cada homogeneizado, completando com $100 \mathrm{~mL}$ de água destilada e $0,3 \mathrm{~mL}$ de solução de fenolftaleína. Sobre esta, foi realizada a titulação com Hidróxido de Sódio 0,1 M, sob agitação constante. Determinações foram realizadas em sextuplicata e resultados expressos em percentual de ácido tartárico em $100 \mathrm{~g}$ de amostra, conforme método 312/IV do INSTITUTO ADOLFO LUTZ (2005).

\section{Avaliação de propriedade mecânica}

A firmeza das bagas de uva foi determinada através de análise de Resistência à Perfuração (punctura) no texturômetro marca Stable Micro Systems, modelo TA-XT2i (Godalming, Inglaterra). Foram considerados os parâmetros Força Máxima até o Rompimento (FMR), e Distância Máxima até o Rompimento (DMR). Foi utilizado probe cilíndrico de alumínio de $2 \mathrm{~mm}$ de diâmetro $(\mathrm{P} / 2)$ em um único 
ciclo. O equipamento foi ajustado para trabalhar nas seguintes condições: velocidade inicial $1 \mathrm{~mm} / \mathrm{s}$, velocidade de teste $1 \mathrm{~mm} / \mathrm{s}$, velocidade pós-teste $1 \mathrm{~mm} / \mathrm{s}$, distância percorrida após contato com a amostra $6 \mathrm{~mm}$, força $5 \mathrm{~g}$, aquisição de dados 200 pps.

As amostras, oito bagas de cada tratamento, foram posicionadas com seu eixo longitudinal na posição horizontal, mantendo sempre o pedúnculo à direita, conforme metodologia proposta por Letaief e colaboradores (2008). Considerou-se um único ponto de punctura para todas as bagas, sendo este o ponto mais elevado na região mediana da baga.

\section{Tratamento estatístico}

Os dados foram tratados estatisticamente através do teste de Kruskal-Wallis. Este teste é indicado para comparar três ou mais tratamentos e é um teste não paramétrico equivalente à ANOVA. Além disto, em alguns casos, foram ajustados modelos e feito o teste de aleatorização utilizando o programa Estatistics.

\section{RESULTADOS E DISCUSSÃO}

\section{Diâmetro e comprimento das bagas}

O comprimento das bagas variou de 2,485 a $3,055 \mathrm{~cm}$, e o diâmetro variou entre $1,700 \mathrm{e} 2,070 \mathrm{~cm}$. Diante do diâmetro médio da baga, aproximadamente $1,8 \mathrm{~cm}$, foi estipulada a profundidade a ser alcançada pelo probe nas análises de textura, sendo fixada em $0,6 \mathrm{~cm}$ ( $1 / 3$ do diâmetro do corpo de prova).

\section{Perda de massa}

A perda de massa das bagas de uva apresentou comportamento linear no intervalo em que ocorreram as análises, com valores de R-quadrado superiores a $0,990 \mathrm{em}$ todos os tratamentos. A maior perda de massa foi registrada no tratamento-controle (X) onde foi notado $20,52 \%$ de perda acumulada de massa fresca. Entre as uvas que receberam cobertura, foi percebida perda de massa acumulada média de $14,73 \%$ ( $0,59 \%$ ao dia), sendo a maior perda registrada no tratamento $\mathrm{H}, 16,45 \%$, e a menor no tratamento B, $12,89 \%$. A perda de massa acumulada dos 13 tratamentos é apresentada na Tabela 2.

Após 25 dias de aplicação da cobertura, foi percebida a diminuição da perda de massa em $56 \%$ (ganho de nove dias), quando comparado o tratamento B em relação ao controle (tratamento X).

Mattiuz e colaboradores (2004), em trabalho com uvas apirênicas da cultivar BRS Morena e da Seleção ${ }^{\circ} 08$ minimamente processadas e armazenadas em bandejas de PET com tampa a $2,5 \pm 1{ }^{\circ} \mathrm{C}$, por 24 dias, obtiveram cerca de $0,16 \%$ de perda de massa fresca acumulada. Albertini e colaboradores (2009), trabalhando com uva Itália armazenada em sacolas de polietileno à temperatura ambiente $\left(21,2 \pm 1,6^{\circ} \mathrm{C}\right)$, por 9 dias, observaram maior percentual de perda de massa, igual a $0,73 \%$ por dia. Mattiuz e colaboradores (2009), em trabalho com três variedades de uvas apirênicas armazenadas em bandejas de PET com tampa, sob duas distintas temperaturas, 12 e $24{ }^{\circ} \mathrm{C}$, obtiveram perdas acumuladas de massa fresca variando entre 0,5 e $2 \%$ ao final de 12 dias.

Na presente pesquisa, as uvas cobertas foram submetidas a um processamento mais severo que as uvas minimamente processadas no trabalho de Mattiuz et al. (2004), registrando perda de massa diária média, em torno de $0,59 \%$ nas uvas tratadas, perfazendo perda acumulada de $14,73 \%$ ao final de 25 dias, mostrando-se 92 vezes superior à observada no referido trabalho, $0,16 \%$ ao final de 24 dias. Aparentemente, a aplicação das coberturas modificou a atmosfera no interior dos frutos. As diferenças nos processos metabólicos característicos de cada cultivar aliada aos efeitos conjugados da temperatura de armazenamento e à embalagem utilizada podem ter contribuído para essa diferença.

Impressão global e análise de cor

Fatores como variedade, maturação e tipo de processamento têm papel fundamental na cor apresentada pelo produto após o processamento (MATTIUZ et al., 2009). O percentual de frutooligossacarídeo incorporado nas formulações A, E e I, $30 \% \mathrm{p} / \mathrm{v}$, conferiram aspecto áspero às bagas de uva, percebido visualmente e através do tato. A incorporação desta concentração do prebiótico na formulação líquida apresentou-se ideal, porém ao secar na superfície da baga, com a perda de água, ocorreu a saturação do soluto e sua consequente precipitação. As demais bagas apresentaram-se lisas, com brilho alterado e com adesividade aumentada, principalmente nas bagas que receberam formulações com 20 ou $30 \%$ de FOS. Os tratamentos D, H e L não apresentaram adesividade. Esta alteração pode ser devida à presença do FOS.

Os valores medianos dos componentes de cor $\mathrm{L}^{*}, \mathrm{a}^{*} \mathrm{e} \mathrm{b*}$, no dia seguinte à aplicação das coberturas comestíveis, estão apresentadas na Tabela 3.

O tratamento-controle apresentou valores intermediários para os três componentes de cor. Para o componente $\mathrm{L}^{*}$, que se relaciona ao brilho, os valores variaram entre 40,66 (tratamento C) e 44,90 (tratamento I). Apenas os tratamentos C, E e $\mathrm{K}$ apresentaram valores inferiores ao tratamentocontrole $(41,75)$, do que se infere que a aplicação das coberturas aumentou o brilho nos outros nove tratamentos.

Para o componente colorimétrico $\mathrm{a}^{*}$, que está 
relacionado à tonalidade de cor, variando do verde $(-a)$ ao vermelho $(+a)$, os valores variaram de $-7,12$ (tratamento F) a -5,80 (tratamento A). Os tratamentos $\mathrm{F}, \mathrm{I}, \mathrm{K}$ e L apresentaram valores inferiores ao controle (-6,71). A aplicação das coberturas não influenciou na tonalidade de cor verde das amostras.

Para o componente colorimétrico $b^{*}$, que está relacionado à tonalidade de cor, variando do azul (-b) ao amarelo $(+b)$, os valores variaram de 11,76 (tratamento D) a 15,09 (tratamento F). Seis tratamentos apresentaram valores para $b^{*}$ inferiores ao controle (X) e seis apresentaram valores superiores, demonstrando que a aplicação das coberturas não exerceu influência neste componente colorimétrico.

\section{Propriedades Físico-Químicas Sólidos Solúveis}

Embora tenha sido notado sutil aumento dos valores de SS durante o armazenamento, não foi percebida linearidade entre estes valores em nenhum dos tratamentos, conforme apresentado na Tabela 4 , indicando assim que as bagas mantiveram boa qualidade química e visual durante todo o período.

Comportamento similar foi observado em outros trabalhos com uvas, em que foram observados valores médios de SS oscilando em diferentes dias de análises para um mesmo tratamento. Foram observados valores médios de SS variando de 10,3 a $17^{\circ}$ Brix em uvas com cobertura à base de quitosana e óleo essencial de orégano (SANTOS et al., 2012), 18 a $21,5^{\circ}$ Brix em uvas cobertas com quitosana e hidroxipropilmetilcelulose com óleo de bergamota (SÁNCHEZ-GONZÁLEZ et al., 2011) e 11,65 a $13,24{ }^{\circ}$ Brix em uvas minimamente processadas que não receberam coberturas (MATTIUZ et al., 2009).

Os resultados relativos aos teores de SS das uvas avaliadas no presente estudo corroboram os resultados obtidos por Pastor e colaboradores (2011), que trabalhando com uvas de mesa variedade Muscatel revestidas por cobertura comestível à base de hidroxipropilmetilcelulose, contendo extrato de própolis, armazenadas sob refrigeração $\left(1-2{ }^{\circ} \mathrm{C}\right)$, não detectaram efeito das coberturas sobre o teor de sólidos solúveis das amostras. A variabilidade natural dos frutos, provavelmente, dificultou a observação de pequenas diferenças que, possivelmente, poderiam ser induzidas pelos tratamentos (PASTOR et al., 2011).

Os valores medianos de $\mathrm{SS}$ dos 13 tratamentos avaliados oscilaram de 17,4 a $23,8^{\circ}$ Brix no intervalo dos 19 dias de análises. Estes valores mostramse satisfatórios para a comercialização de uvas apirênicas nos mercados Brasileiro e Internacional.
De acordo com Mascarenhas et al. (2010), uvas de mesa com teores de SS igual ou superior a 15\% são consideradas aptas para comercialização na região Nordeste do Brasil. Outro valor considerado como limite mínimo para SS é citado por Mattiuz et al. (2009), que atribuíram o teor mínimo de $14^{\circ}$ Brix para atendimento às normas internacionais de comercialização para as uvas de mesa.

No primeiro dia de análise, os valores variaram entre 17,4 (tratamento $\mathrm{X}$ ) e $20,2^{\circ}$ Brix (tratamento $\mathrm{K}$ ), enquanto no último dia de análise, variaram entre 19,6 (tratamento X) e 23,8 ${ }^{\circ}$ Brix. O tratamento $\mathrm{X}$ registrou menores valores de $\mathrm{SS}$ em três dos sete dias analisados, que pode estar relacionado com a ausência de aditivos em sua composição, tanto de cera de carnaúba quanto de FOS.

Os tratamentos A, E e I, que tinham na composição da formulação $30 \%$ de FOS, não apresentaram diferenças significativas com relação a outros tratamentos. Aparentemente, a alta concentração de FOS não interferiu no aumento do valor de SS destas amostras.

\section{Acidez total titulável}

Durante o processo natural de maturação das frutas, os ácidos são metabolizados a açúcares. Assim, observa-se aumento do teor de sólidos solúveis e a diminuição do teor de acidez. Observaram-se baixos valores para ATT na maioria das leituras, de 0,58 a $0,85 \%$ de ácido tartárico, conforme apresentado na Tabela 5 .

Não foi observada linearidade nas leituras dos valores de ATT. Comportamento similar foi observado nos trabalhos com uvas experimentados por Santos et al. (2012) e Mattiuz et al. (2009).

No primeiro dia de análise, os valores de ATT variaram entre 0,63 (tratamento A) e 0,76\% (tratamento X), conforme apresentado na Tabela 5. Os menores valores de ATT foram registrados nos tratamentos $\mathrm{K}$ e L, concentrando, nestes, cinco das sete menores medianas registradas. O último dia de análise oscilou entre 0,63 (tratamento F) e $0,85 \%$ (tratamento X).

O máximo valor de ATT obtido foi de $0,85 \%$, o que demonstra que todos os tratamentos se encontravam em condições de comercialização nos mercados Brasileiro e Internacional durante os 19 dias em que ocorreram as análises (MASCARENHAS et al., 2010; MATTIUZ et al., 2004).

\section{titulável}

Relação Sólidos solúveis/Acidez total

A relação $\mathrm{SS} / \mathrm{ATT}$, um dos principais parâmetros fisico-quimicos indicativos da qualidade de uvas de mesa, variou entre 22,90 e 31,37 no primeiro dia de análise, mantendo esta grande 
amplitude entre os valores durante os sete dias analisados, tendo valores finais variando de 23,24 a 37,86 no $19^{\circ}$ dia após a aplicação das coberturas, conforme apresentado na Tabela 6. Esta grande amplitude dos valores obtidos está de acordo com a variação obervada nos valores de SS e ATT.

Observa-se que a totalidade dos valores da relação SS/ATT mostrou-se superior a 22,00. De acordo com Mascarenhas e colaboradores (2010), é desejável que esta relação seja superior a 20. Sendo assim, todos os 13 tratamentos avaliados apresentaram-se dentro dos padrões físicoquímicos mínimos para comercialização no mercado internacional.

Os valores obtidos para os parâmetros físico-químicos SS, ATT e SS/ATT não diferiram entre as amostras tratadas e a amostra-controle. Todos os tratamentos mostraram-se dentro dos padrões internacionais de comercialização, com SS acima de $14{ }^{\circ}$ Brix, ATT abaixo de 1,5\% de ácido tartárico/100 g de amostra e relação SS/ATT superior a 20 (MASCARENHAS et al., 2010; MATTIUZ et al., 2004; MATTIUZ et al., 2009).

\section{Propriedade Mecânica}

Força Máxima até o Rompimento (FMR)

Durante o armazenamento, percebeu-se que o tratamento $\mathrm{A}$ foi o único que registrou, em dois momentos, a maior FMR, ocorrendo no $4^{\circ} \mathrm{e}$ $19^{\circ}$ dias após a aplicação das coberturas, conforme apresentado na Tabela 7. A menor FMR repetiuse apenas no tratamento $\mathrm{G}$, no $4^{\circ}$ e $7^{\circ}$ dias após a aplicação das coberturas.

A amostra-controle, tratamento $\mathrm{X}$, obteve valores intermediários de FMR ao longo dos dias de análise, indicando que a aplicação das coberturas comestíveis não interferiu neste parâmetro de textura, não exercendo influência na resistência mecânica da superfície da baga.

Distância Máxima até o Rompimento (DMR)

Os valores medianos do parâmetro de textura DMR aumentaram durante o armazenamento em todos os tratamentos, conforme apresentado na Tabela 8. Aparentemente, existe efeito do tempo sobre a DMR para todos os tratamentos.

$\mathrm{O}$ tratamento $\mathrm{X}$ registrou valor de DMR entre os três maiores, em seis dos sete momentos de análise ( $86 \%$ dos dias). Tal fato pode ser atribuído à perda de massa das amostras, decorrente da respiração dos frutos durante o armazenamento, que foi nitidamente maior no tratamento X. Esta perda de massa causa diminuição do turgor das bagas. Ao aplicar as forças para punção, a epiderme do fruto oferece mais resistência porque está mais flexível e tem maior capacidade de deformação antes da ruptura (CARVALHO FILHO et al., 2005). Esse fenômeno foi notado por Drake e Fellman (1987),que encontraram aumento inexplicável na resistência mecânica, sendo este atribuído à perda de água nos frutos.

TABELA 1 - Composição das formulações das coberturas comestíveis para aplicação em uvas Thompson.

\begin{tabular}{|c|c|c|c|c|c|}
\hline & & \multicolumn{4}{|c|}{ Concentração de CERA } \\
\hline & & $50 \%$ & $33 \%$ & $25 \%$ & $0 \%$ \\
\hline \multirow{4}{*}{ 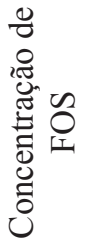 } & $30 \%$ & A & $\mathrm{E}$ & I & FNFF \\
\hline & $20 \%$ & B & $\mathrm{F}$ & $\mathrm{J}$ & FNFF \\
\hline & $10 \%$ & $\mathrm{C}$ & G & $\mathrm{K}$ & FNFF \\
\hline & $0 \%$ & $\mathrm{D}$ & $\mathrm{H}$ & $\mathrm{L}$ & $\mathrm{X}$ \\
\hline
\end{tabular}

FNFF - Formulação não formadora de filme 
TABELA 2 - Perda de massa acumulada em uvas Thompson após aplicação de coberturas comestíveis e acondicionadas sob refrigeração por 25 dias (\%).

\begin{tabular}{|c|c|c|c|c|c|c|c|c|c|}
\hline \multirow{2}{*}{ Tratamento } & \multicolumn{9}{|c|}{ Dias após aplicação de cobertura } \\
\hline & 1 & 4 & 7 & 10 & 13 & 16 & 19 & 22 & 25 \\
\hline A & 0,00 & 2,82 & 4,54 & 6,07 & 7,42 & 9,75 & 11,91 & 13,35 & 15,17 \\
\hline $\mathrm{B}$ & 0,00 & 1,29 & 2,98 & 4,58 & 6,01 & 7,52 & 9,11 & 10,90 & 12,89 \\
\hline $\mathrm{C}$ & 0,00 & 1,89 & 3,56 & 5,19 & 7,31 & 9,22 & 11,34 & 12,93 & 15,00 \\
\hline $\mathrm{D}$ & 0,00 & 1,80 & 3,52 & 5,25 & 7,01 & 8,89 & 11,27 & 13,25 & 14,76 \\
\hline $\mathrm{E}$ & 0,00 & 2,31 & 4,14 & 5,62 & 7,18 & 9,01 & 10,60 & 12,31 & 14,77 \\
\hline $\mathrm{F}$ & 0,00 & 2,30 & 4,22 & 5,84 & 7,44 & 9,55 & 11,31 & 13,11 & 14,80 \\
\hline $\mathrm{G}$ & 0,00 & 2,30 & 3,91 & 5,60 & 7,22 & 8,96 & 10,87 & 12,64 & 14,65 \\
\hline $\mathrm{H}$ & 0,00 & 2,03 & 4,42 & 6,46 & 8,31 & 10,39 & 12,55 & 14,47 & 16,45 \\
\hline $\mathrm{I}$ & 0,00 & 1,75 & 3,97 & 6,10 & 7,96 & 9,91 & 12,58 & 14,20 & 16,19 \\
\hline $\mathrm{J}$ & 0,00 & 2,27 & 3,68 & 4,97 & 6,89 & 8,54 & 10,21 & 11,95 & 14,10 \\
\hline $\mathrm{K}$ & 0,00 & 1,90 & 3,60 & 5,21 & 6,72 & 8,43 & 10,43 & 11,95 & 14,62 \\
\hline $\mathrm{L}$ & 0,00 & 1,94 & 3,69 & 5,36 & 6,72 & 8,38 & 10,18 & 11,88 & 13,37 \\
\hline X & 0,00 & 2,73 & 4,86 & 7,29 & 9,94 & 12,50 & 15,20 & 17,66 & 20,52 \\
\hline
\end{tabular}

TABELA 3 - Componentes colorimétricos L*, a* e b* de uvas Thompson após aplicação de coberturas comestíveis.

\begin{tabular}{cccc}
\hline \multirow{2}{*}{ Tratamento } & \multicolumn{3}{c}{ Componente colorimétrico } \\
& $\mathrm{L}^{*}$ & $\mathrm{a}^{*}$ & $\mathrm{~b}^{*}$ \\
\hline A & 43,25 & $-5,80$ & 13,47 \\
B & 41,79 & $-6,01$ & 13,04 \\
C & 40,66 & $-6,17$ & 14,50 \\
D & 42,18 & $-5,93$ & 11,76 \\
E & 40,83 & $-6,70$ & 12,76 \\
F & 43,73 & $-7,12$ & 15,09 \\
G & 43,32 & $-6,38$ & 12,74 \\
H & 43,94 & $-6,35$ & 13,91 \\
I & 44,90 & $-7,03$ & 14,98 \\
J & 42,11 & $-6,30$ & 12,38 \\
K & 41,13 & $-6,82$ & 13,59 \\
L & 42,09 & $-6,84$ & 13,10 \\
X & 41,75 & $-6,71$ & 13,14 \\
\hline
\end{tabular}

TABELA 4 - Sólidos Solúveis de uvas Thompson após aplicação de coberturas comestíveis e acondicionadas sob refrigeração por 19 dias ( ${ }^{\circ}$ Brix).

\begin{tabular}{cccccccc}
\hline \multirow{2}{*}{ Tratamento } & \multicolumn{7}{c}{ Dias após aplicação de cobertura } \\
& 1 & 4 & 7 & 10 & 13 & 16 & 19 \\
\hline A & 19,60 & 20,80 & 19,90 & 20,80 & 19,80 & 21,00 & 22,20 \\
B & 18,40 & 19,20 & 20,40 & 19,00 & 20,20 & 21,80 & 20,40 \\
C & 17,80 & 19,10 & 18,70 & 19,60 & 20,20 & 19,80 & 21,00 \\
D & 19,00 & 19,60 & 20,40 & 20,20 & 21,00 & 20,40 & 21,00 \\
E & 19,00 & 20,00 & 20,80 & 19,00 & 21,20 & 20,80 & 21,20 \\
F & 19,00 & 20,00 & 20,00 & 20,20 & 23,00 & 22,20 & 23,20 \\
G & 18,60 & 18,00 & 20,80 & 20,40 & 20,00 & 19,80 & 22,40 \\
H & 19,00 & 18,00 & 21,00 & 20,20 & 19,40 & 20,00 & 20,40 \\
I & 19,00 & 19,60 & 20,40 & 20,00 & 20,40 & 18,20 & 23,00 \\
J & 20,10 & 20,80 & 21,80 & 22,00 & 21,60 & 22,60 & 23,20 \\
K & 20,20 & 20,40 & 21,60 & 21,40 & 22,40 & 23,00 & 22,60 \\
L & 19,20 & 20,00 & 21,20 & 20,80 & 21,00 & 21,80 & 23,80 \\
X & 17,40 & 20,60 & 21,00 & 22,00 & 19,40 & 19,80 & 19,60 \\
\hline
\end{tabular}


TABELA 5 -Acidez Total Titulável de uvas Thompson após aplicação de coberturas comestíveis e acondicionadas sob refrigeração por 19 dias (\% de ácido tartárico).

\begin{tabular}{cccccccc}
\hline \multirow{2}{*}{ Tratamento } & \multicolumn{7}{c}{ Dias após aplicação de cobertura } \\
& 1 & 4 & 7 & 10 & 13 & 16 & 19 \\
\hline A & 0,62 & 0,64 & 0,71 & 0,64 & 0,68 & 0,70 & 0,69 \\
B & 0,65 & 0,67 & 0,70 & 0,71 & 0,73 & 0,65 & 0,66 \\
C & 0,66 & 0,67 & 0,70 & 0,62 & 0,68 & 0,67 & 0,73 \\
D & 0,66 & 0,65 & 0,72 & 0,67 & 0,73 & 0,69 & 0,71 \\
E & 0,68 & 0,70 & 0,69 & 0,64 & 0,68 & 0,70 & 0,70 \\
F & 0,65 & 0,63 & 0,72 & 0,62 & 0,65 & 0,63 & 0,63 \\
G & 0,72 & 0,68 & 0,61 & 0,62 & 0,70 & 0,69 & 0,69 \\
H & 0,71 & 0,72 & 0,65 & 0,65 & 0,70 & 0,71 & 0,67 \\
I & 0,68 & 0,65 & 0,68 & 0,67 & 0,74 & 0,81 & 0,69 \\
J & 0,71 & 0,70 & 0,66 & 0,71 & 0,71 & 0,70 & 0,68 \\
K & 0,66 & 0,60 & 0,59 & 0,58 & 0,75 & 0,64 & 0,74 \\
L & 0,64 & 0,61 & 0,60 & 0,61 & 0,64 & 0,58 & 0,63 \\
X & 0,76 & 0,63 & 0,59 & 0,62 & 0,71 & 0,74 & 0,84 \\
\hline
\end{tabular}

TABELA 6 - Relação Sólidos Solúveis/Acidez Total Titulável de uvas Thompson após aplicação de coberturas comestíveis e acondicionadas sob refrigeração por 19 dias.

\begin{tabular}{cccccccc}
\hline \multirow{2}{*}{ Tratamento } & \multicolumn{7}{c}{ Dias após aplicação de cobertura } \\
& 1 & 4 & 7 & 10 & 13 & 16 & 19 \\
\hline A & 31,37 & 32,67 & 28,11 & 32,64 & 29,14 & 29,84 & 32,32 \\
B & 28,44 & 28,83 & 29,24 & 26,92 & 27,65 & 33,31 & 30,95 \\
C & 26,86 & 28,61 & 26,81 & 31,46 & 29,59 & 29,34 & 28,66 \\
D & 28,60 & 30,28 & 28,32 & 30,33 & 28,63 & 29,59 & 29,57 \\
E & 28,13 & 28,65 & 30,25 & 29,55 & 31,04 & 29,90 & 30,25 \\
F & 29,43 & 31,64 & 27,59 & 32,84 & 35,18 & 35,40 & 37,05 \\
G & 25,96 & 26,53 & 33,86 & 32,96 & 28,42 & 28,80 & 32,70 \\
H & 26,83 & 25,15 & 32,49 & 31,00 & 27,65 & 28,33 & 30,33 \\
I & 27,89 & 30,11 & 30,08 & 29,81 & 27,55 & 22,51 & 33,45 \\
J & 28,34 & 29,63 & 33,12 & 31,12 & 30,32 & 32,50 & 34,21 \\
K & 30,44 & 33,75 & 36,84 & 36,96 & 30,03 & 36,08 & 30,44 \\
L & 30,00 & 32,52 & 35,59 & 34,14 & 32,65 & 37,85 & 37,86 \\
X & 22,90 & 32,84 & 35,47 & 35,38 & 27,36 & 26,79 & 23,24 \\
\hline
\end{tabular}

TABELA 7 - Força Máxima até o Rompimento de uvas Thompson após aplicação de coberturas comestíveis e acondicionadas sob refrigeração por 19 dias.

\begin{tabular}{cccccccc}
\hline \multirow{2}{*}{ Tratamento } & \multicolumn{7}{c}{ Dias após aplicação de cobertura } \\
& 1 & 4 & 7 & 10 & 13 & 16 & 19 \\
\hline A & 294,85 & 337,50 & 307,50 & 314,70 & 322,00 & 317,35 & 343,75 \\
B & 291,90 & 307,60 & 286,05 & 361,60 & 256,10 & 344,55 & 310,65 \\
C & 265,90 & 282,00 & 353,20 & 312,95 & 319,95 & 326,25 & 316,85 \\
D & 320,55 & 327,55 & 358,80 & 320,65 & 302,05 & 298,40 & 286,70 \\
E & 276,05 & 273,20 & 316,20 & 289,60 & 315,60 & 285,65 & 305,40 \\
F & 310,75 & 324,85 & 281,45 & 297,95 & 334,60 & 265,55 & 298,00 \\
G & 340,60 & 199,60 & 193,95 & 267,55 & 298,80 & 307,10 & 293,45 \\
H & 336,10 & 300,80 & 302,70 & 304,50 & 284,45 & 266,40 & 256,10 \\
I & 284,20 & 315,50 & 300,60 & 302,40 & 264,00 & 351,45 & 311,05 \\
J & 288,42 & 247,75 & 295,95 & 282,60 & 343,10 & 305,05 & 295,15 \\
K & 270,45 & 223,30 & 299,00 & 233,00 & 290,25 & 306,80 & 313,30 \\
L & 303,00 & 236,00 & 280,60 & 315,95 & 253,45 & 313,10 & 290,95 \\
X & 279,45 & 233,00 & 346,95 & 307,05 & 312,35 & 243,50 & 281,35 \\
\hline
\end{tabular}


TABELA 8 - Distância Máxima até o Rompimento de uvas Thompson após aplicação de coberturas comestíveis e acondicionadas sob refrigeração por 19 dias.

\begin{tabular}{cccccccc}
\hline \multirow{2}{*}{ Tratamento } & \multicolumn{7}{c}{ Dias após aplicação de cobertura } \\
& 1 & 4 & 7 & 10 & 13 & 16 & 19 \\
\hline A & 2,95 & 3,60 & 3,75 & 3,90 & 4,40 & 4,55 & 4,20 \\
B & 2,85 & 3,25 & 3,60 & 4,30 & 4,00 & 4,30 & 4,30 \\
C & 2,85 & 3,75 & 4,30 & 3,70 & 4,55 & 4,30 & 4,90 \\
D & 2,95 & 3,55 & 3,35 & 3,80 & 3,90 & 4,05 & 4,10 \\
E & 2,90 & 3,40 & 3,50 & 4,10 & 3,85 & 4,55 & 4,40 \\
F & 3,35 & 3,25 & 3,50 & 3,45 & 4,15 & 4,15 & 4,25 \\
G & 3,20 & 4,50 & 4,90 & 5,05 & 4,65 & 4,30 & 4,40 \\
H & 2,90 & 3,70 & 3,35 & 3,70 & 3,85 & 3,95 & 4,05 \\
I & 3,75 & 3,60 & 3,50 & 3,95 & 4,05 & 4,50 & 4,70 \\
J & 3,10 & 3,20 & 3,65 & 3,90 & 4,15 & 4,00 & 4,30 \\
K & 3,30 & 4,00 & 4,75 & 4,10 & 3,55 & 4,15 & 4,40 \\
L & 2,90 & 4,20 & 4,15 & 3,85 & 3,70 & 4,05 & 3,85 \\
X & 3,60 & 4,30 & 4,50 & 3,80 & 4,85 & 4,85 & 5,10 \\
\hline
\end{tabular}

\section{CONCLUSÃO}

Amostras tratadas com cera de carnaúba adicionada de FOS mantêm valores de SS, ATT, relação SS/ATT, propriedades mecânicas e componentes colorimétricos $\mathrm{L}^{*}, \mathrm{a}^{*} \mathrm{e} \mathrm{b}^{*}$ dentro dos padrões de comercialização. As uvas Thompson tratadas e adicionadas de FOS apresentam aumento de até $56 \%$ no tempo de vida de prateleira, quando comparadas com o controle, sendo a formulação B ( $50 \%$ de cera, $20 \%$ de FOS) a que apresenta melhor potencial de uso comercial, seguida das formulações K $(25 \%$ de cera, $10 \%$ de FOS $)$ e L $(25 \%$ de cera, $0 \%$ de FOS).

\section{REFERÊNCIAS}

ALBERTINI, S.; MIGUEL, A. C. A.; SPOTO, M. H. F. Influência de sanificantes nas características físicas e químicas de uva Itália. Ciência Tecnologia de Alimentos, Campinas, v. 29, n.3, p.504-507, 2009.

ALLEONI, A. C.; JACOMINO, A. P.; ROSA, A. S. Recobrimento de laranja 'Pêra' com filme de concentrado proteico de soro de leite associado a plastificantes. Pesquisa Agropecuária Brasileira, Brasília, v.41, n.8, p.1221-1226, 2006.

BALDWIN, E.A.; HAGENMAIER, R. D. Introduction. In: BALDWIN, E.A.; HAGENMAIER, R. D.; BAI, J. (Ed.). Edible coating and films to improve food quality. $2^{\text {nd }}$ ed. Boca Raton: Taylor \& Francis Group, LLC, 2011. p.1-12.
BERTÉ, K. A. S.; IZIDORO, D. R.; DUTRA, F. L. G.; HOFFMANN-RIBANI, R. Desenvolvimento de gelatina funcional de erva-mate. Ciência Rural, Santa Maria, v.41, n.2, p.354-360, 2011. Disponível em: $<$ http://www.scielo.br/scielo.php?script $=$ sci arttext\&pid $=$ S0103-84782011000200029\&lng $=$ en

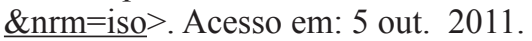

CARVALHO FILHO, C. D.; HONORIO, S. L.; GIL, J. M. Propriedades mecânicas de cerejas (Prunus avium L.), cv. Ambrunés, Cobertas com emulsão de cera de carnaúba e zeína. Boletim CEPPA, Curitiba, v.23, n.1, p.23-36,2005.

CARVALHO FILHO, C. D.; HONORIO, S. L.; GIL, J. M. Qualidade pós-colheita de cerejas cv. Ambrunés utilizando coberturas comestíveis. Revista Brasileira de Fruticultura, Jaboticabal, v.28, n.2, p.180-184, 2006.

DEL NOBILE, M. A.; CONTE, A.; SCROCCO, C.; BRESCIA, I.; SPERANZA, B.; SINIGAGLIA, M.; PERNIOLA, R.; ANTONACCI, D. A study on quality loss minimally processed grapes as affected by film packaging. Postharvest Biology and Technology, New York, v.51, p.21-26, 2009.

DRAKE, S. R.; FELLMANN, J. K. Indicators of maturity and storage quality of "Rainier Sweet" Cherry. Hortscience, Alexandria, v.22, n.2, p. 283285, 1987.

FAKHOURI, F.M.; FONTES, L.C.B.; GONÇALVES, P. V. de M.; MILANEZ, C. R.; STEEL, C. J.; COLLARES-QUEIROZ, F. P. Filmes e coberturas comestíveis compostas à base de amidos nativos e gelatina na conservação e aceitação sensorial de uvas Crimson. Ciência Tecnologia de Alimentos, Campinas, v.27, n.2, p. 369-375, 2007. 
HARTEMINK, R.; VAN LAERE, K.M.J.; ROMBOUTS, F.M. Growth of enterobacteria on fructo-oligosaccharides. Journal of Applied Microbiology, Wageningnen, v. 383, p.367$374,1997$.

INSTITUTO ADOLPHO LUTZ. Métodos físicoquímicos para análise de alimentos. 4. ed. São Paulo: Instituto Adolfo Lutz, 2005. 1020p.

LETAIEF, H.; ROLLE, L.; ZEPPA, G.; GERBI, V. Assessment of grape skin hardness by a puncture test. Journal of the Science and Food Agriculture, London, v.88, p.1567-1575, 2008.

LIMA, M.A.C. Cultivo da videira. Petrolina: Embrapa Semi-Árido, 2010. (Sistemas de Produção, 1).

LULU, J.; CASTRO, J. V., PEDRO JÚNIOR, M. J. Efeito do microclima na qualidade da uva de mesa 'Romana' (A 1105) cultivada sob cobertura plástica. Revista Brasileira de Fruticultura, Jaboticabal, v.27, n.3, p.422-425, 2005.

MASCARENHAS, R. J.; SILVA, S. D.; LOPES, J. D.; LIMA, M. A. C. Avaliação sensorial de uvas de mesa produzidas no Vale do São Francisco e comercializadas em João Pessoa - PB. . Revista Brasileira de Fruticultura, Jaboticabal, v.32, n.4, p.993-1000, 2010.

MATTIUZ, B.; MigUEL, A. C. A.; GALATI, V. C.; NACHTIGAL, J. C. Efeito da temperatura no armazenamento de uvas apirênicas minimamente processadas. Revista Brasileira de Fruticultura, Jaboticabal, v.31, n.1, p.44-52, 2009.

MATTIUZ, B.; MIGUEL, A. C. A.; NACHTIGAL, J. F. D.; CAMARGO, U. A. Processamento mínimo de uvas de mesa sem semente. . Revista Brasileira de Fruticultura, Jaboticabal, v.26, n.2, p.226-229, 2004.

MELLO, L.M.R. Atuação do Brasil no mercado vitivinícola mundial: panorama 2010. Bento Gonçalves: Embrapa Uva e Vinho. 2011a. Versão eletrônica.

MELLO, L.M.R. Vitivinicultura brasileira: panorama 2010. Bento Gonçalves: Embrapa Uva e Vinho. 2011b. Versão eletrônica.
MOTA, W. F.; SALOMÃO, L. C. C.; NERES, C. R. L.; MIZOBUTSI, G. P.; NEVES, L. L. de M. Uso de cera de carnaúba e saco plástico poliolefínico na conservação pós-colheita do maracujáamarelo. Revista Brasileira de Fruticultura, Jaboticabal, v.28, n.2, p.190-193, 2006.

PASSOS, L. M.L.; PARK, Y.K. Frutooligossacarídeos: Implicações na saúde humana e utilização em alimentos. Ciência Rural, Santa Maria, v.33, n.2, 2003.

PASTOR, C.; SÁNCHEZ-GONZÁLEZ, L.; MARCILLA, A.; CHIRALT, A.; CHÁFER, M.; GONZÁLEZ-MARTÍNEZ, C. Quality and safety of table grapes coated with hydroxypropylmethylcellulose edible coatings containing própolis extract. Postharvest Biology and Technology, Amsterdam, v.60, p.64-70, 2011.

ROBERFROID, M.; GIBSON, G.R.; HOYLES, L.; et al. Prebiotic effects: metabolic and health benefits. British Journal of Nutrition, Cambridge, v. 104, sup. S2, 2010. Disponível em: $\leq$ http://journals.cambridge. org/download.php? file=\%2FBJN\%2FBJN104 $\underline{\mathrm{S} 2 \% 2 \mathrm{FS} 0007114510003363 \mathrm{a} . \mathrm{pdf} \& \mathrm{code}=\mathrm{ec} 00 \mathrm{~d} 4 \mathrm{c}}$ 6b45f667967d2aaa56a4c8e16>. Acesso em: 4 out. 2011.

SÁNCHEZ-GONZÁLEZ,L;PASTOR,C.; VARGAS, M.; CHIRALT, A.; GONZÁLEZ-MARTÍNEZ, C. Effect of hydroxypropylmethylcellulose and chitosan coatings with and whithout bergamot essential oil on quality and safety of cold-stored grapes. Postharvest Biology and Technology, New York, v.60, p.57-63, 2011.

SANTOS, N. S. T.; AGUIAR, A. J.A. A.; OLIVEIRA, C. E. V.; SALES, C. V.; SILVA, S. M.; SILVA, R. S.; STAMFORD, T. C. M; SOUZA, E. L. Efficacy of application of a coating composed of chitosan and Origanum vulgare L. essential oil to control Rhizopus stolonifer and Aspergilus niger in grapes (Vitis labrusca L.). Food Microbiology, Cambridge, v.32, p.345-353, 2012.

VU, K. D.; HOLLINGSWORTH, R. G.; LEROUX, E.; SALMIERI, S., LACROIX, M. Development of edible bioactive coating based on modified chitosan for increasing the shelf life of strawberries. Food Research International, Ottawa, v.44, p.198-203, 2011.

ZHAO, Y. Application of commercial coatings. In: BALDWIN, E.A.; HAGENMAIER, R. D.; BAI, J. (Ed.). Edible coating and films to improve food quality. $2^{\text {nd }}$ ed. Boca Raton: Taylor \& Francis Group, 2011. p.319-331. 